\title{
Consecutive Detecting Arrays for Interaction Faults *
}

\author{
Ce Shi ${ }^{1}$, Ling Jiang ${ }^{2}$ and Aiyuan $\mathrm{Tao}^{3}$ \\ ${ }^{1}$ School of Statistics and Mathematics \\ Shanghai Lixin University of Accounting and Finance, Shanghai 201209, China \\ ${ }^{2}$ College of Information Technology \\ Shanghai Ocean University, Shanghai 201306, China \\ ${ }^{3}$ School of International Economics and Trade \\ Shanghai Lixin University of Accounting and Finance, Shanghai 201209, China
}

May 28, 2019

\begin{abstract}
The concept of detecting arrays was developed to locate and detect interaction faults arising between the factors in a component-based system during software testing. In this paper, we propose a family of consecutive detecting arrays (CDAs) in which the interactions between factors are considered to be ordered. CDAs can be used to generate test suites for locating and detecting interaction faults between neighboring factors. We establish a general criterion for measuring the optimality of CDAs in terms of their size. Based on this optimality criterion, the equivalence between optimum CDAs and consecutive orthogonal arrays with prescribed properties is explored. Using the advantages of this equivalence, a great number of optimum CDAs are presented. In particular, the existence of optimum CDAs with few factors is almost completely determined.
\end{abstract}

Keywords: consecutive detecting arrays; consecutive covering arrays; consecutive orthogonal arrays; optimality; equivalence

Mathematics Subject Classifications (2010): 05B15, 05B20, 62K15, 94C12

\section{Introduction}

Throughout this paper, let $I_{n}$ be the set of the first $n$ positive integers. An $N \times k$ array with entries from an alphabet $\{0,1, \cdots, v-1\}$ of size $v$ is said to be a covering array (CA) (resp. orthogonal array (OA)) if each $N \times t$ subarray contains each $t$-tuple at least (resp. exactly ) $\lambda$ times among its rows. It is denoted by $\mathrm{CA}_{\lambda}(N ; t, k, v)$ (resp. $\left.\mathrm{OA}_{\lambda}(N ; t, k, v)\right)$. When $\lambda=1$, the notation $\mathrm{CA}(N ; t, k, v)$ (resp. $\mathrm{OA}(t, k, v))$ is often used.

CAs are often used to generate test suites in a component-based system, and have various applications in software and hardware, circuit design, and so on. They present a useful alternative to exhaustive testing, and the use of these arrays to construct test suites and their ability to dramatically reduce the testing burden are supported by many empirical results [18, 19]. CAs have been studied extensively, and a great number of methods and results have been reported [3, 6, 8, 9, 10, 13, 16, 17, 23].

\footnotetext{
*Correspondence to: Aiyuan Tao (taoaiyuan@ 126.com). This work was supported by NSFC No. 11301342 and Natural Science Foundation of Shanghai No. 17ZR1419900.
} 
If the selection of the $t$ columns is restricted by considering only consecutive columns, the ensuing problem is closely related to the CA framework. More specifically, Godbole et al. [11] introduced the concept of a consecutive covering array (CCA), in which the structure of the columns captures some linear progression of data (for example, data across a series of consecutive dates) or data organized by consecutive proximity (for example, consecutive switches in a circuit). CCAs (resp. consecutive orthogonal arrays (COAs)), denoted as $\operatorname{CCA}(N ; t, k, v)\left(\right.$ resp. $\left.\mathrm{COA}_{\lambda}(t, k, v)\right)$, are $N \times k$ arrays with entries from a set $V$ of $v$ symbols such that each set of $t$ consecutive columns contains each $t$-tuple at least once (resp. exactly $\lambda$ times) among its rows. The transpose of the following array is a $\operatorname{CCA}(9 ; 2,21,3)$ over $\mathbb{Z}_{3}$.

$$
\left(\begin{array}{lllllllllllllllllllll}
0 & 0 & 0 & 0 & 0 & 0 & 0 & 0 & 0 & 0 & 0 & 0 & 0 & 0 & 0 & 0 & 0 & 0 & 0 & 0 & 0 \\
0 & 1 & 0 & 1 & 0 & 1 & 0 & 1 & 0 & 1 & 0 & 1 & 0 & 1 & 0 & 1 & 0 & 1 & 0 & 1 & 0 \\
0 & 2 & 0 & 2 & 0 & 2 & 0 & 2 & 0 & 2 & 0 & 2 & 0 & 2 & 0 & 2 & 0 & 2 & 0 & 2 & 0 \\
1 & 0 & 1 & 0 & 1 & 0 & 1 & 0 & 1 & 0 & 1 & 0 & 1 & 0 & 1 & 0 & 1 & 0 & 1 & 0 & 1 \\
1 & 1 & 1 & 1 & 1 & 1 & 1 & 1 & 1 & 1 & 1 & 1 & 1 & 1 & 1 & 1 & 1 & 1 & 1 & 1 & 1 \\
1 & 2 & 1 & 2 & 1 & 2 & 1 & 2 & 1 & 2 & 1 & 2 & 1 & 2 & 1 & 2 & 1 & 2 & 1 & 2 & 1 \\
2 & 0 & 2 & 0 & 2 & 0 & 2 & 0 & 2 & 0 & 2 & 0 & 2 & 0 & 2 & 0 & 2 & 0 & 2 & 0 & 2 \\
2 & 1 & 2 & 1 & 2 & 1 & 2 & 1 & 2 & 1 & 2 & 1 & 2 & 1 & 2 & 1 & 2 & 1 & 2 & 1 & 2 \\
2 & 2 & 2 & 2 & 2 & 2 & 2 & 2 & 2 & 2 & 2 & 2 & 2 & 2 & 2 & 2 & 2 & 2 & 2 & 2 & 2
\end{array}\right) .
$$

CCAs represent a similar family to CAs. The analogy between CAs and CCAs is almost obvious. Just as CAs can be used for combinatorial testing, CCAs can be used to generate test suites for combinatorial testing of neighboring factors, such as in circuit testing, signal processing, and so on [25]. Although CAs can also be used to test such software systems, they are larger than CCAs for fixed $t, k, v$. For example, the best upper bound on the size of $\mathrm{CA}(N ; 2,21,3)$ is 16 , but the above example gives us a CCA with only 9 rows. In [11], Godbole et al. focused on $\operatorname{CCA}(N ; t, k, 2)$ using a probabilistic approach based on an appropriate Markov chain method. This allowed them to determine the probability distribution function of a random variable that enumerates the number of uncovered consecutive $t$-subarrays in the case of a $n \times k$ binary array obtained by realizing $k n$ Bernoulli variables. The more general problem of $\operatorname{CCA}(N ; t, k, v)$, i.e., establishing the probability distribution function of the random variable enumerating the uncovered consecutive $t$-subarrays with the Markov chain method, was considered in [12].

When using CAs to generate test suites, the columns of the CA represent factors affecting the response and the entries within columns indicate the settings or values for that factor. The rows then represent the tests to be run. Thus, testing with a CA can indicate the presence or absence of interaction faults for up to $t$ factors. This constitutes a valuable step in the process of screening a system for interaction faults prior to its release. However, the location and magnitude of the interactions causing the faults may be far from clear. In practical terms, tests that reveal the location of the interaction faults are of considerable interest. For this, Colbourn and McClary formalized the problem of the nonadaptive location of interaction faults under the hypothesis that the system contains (at most) $d$ faults, each involving (at most) $t$ interacting factors [7]. They proposed the notion of detecting arrays to solve this problem.

Let $A=\left(a_{i j}\right)\left(i \in I_{N}, j \in I_{k}\right)$ be an $N \times k$ array with entries from an alphabet $V$ of size $v$. Each $t$-set of columns with $t$-tuples of values for those columns is called a $t$-way interaction, denoted by $T=\left\{\left(j_{r}, x_{r}\right): x_{r} \in V, 1 \leq r \leq t\right\}$, where $1 \leq j_{1}<j_{2}<\cdots<j_{t} \leq k$. Write $\rho(A, T)$ for the set of indices of rows of $A$ that cover $T$, i.e., $\rho(A, T)=\left\{i: a_{i j_{r}}=x_{r}, 1 \leq r \leq t\right\}$. For an arbitrary set $\mathcal{T}$ of interactions, define $\rho(A, \mathcal{T})=\cup_{T \in \mathcal{T}} \rho(A, T)$. Furthermore, suppose that $A=\left(a_{i j}\right)\left(i \in I_{N}, j \in I_{k}\right)$ is a 
$\mathrm{CA}(N ; t, k, v)$ over $V$. Write $\mathcal{I}_{t}$ for the set of all $t$-way interactions of $A$. For any $\mathcal{T} \subseteq \mathcal{I}_{t}$ with $|\mathcal{T}|=d$ and any $T \in \mathcal{I}_{t}$, if we have

$$
\rho(A, T) \subseteq \rho(A, \mathcal{T}) \Leftrightarrow T \in \mathcal{T},
$$

then the array $A$ is called a $(d, t)$-detecting array (DA), denoted by $(d, t)-\mathrm{DA}(N ; k, v)$.

Similar to CAs, CCAs can generate test suites for combinatorial testing of neighboring factors to indicate the presence or absence of faulty interactions, they cannot identify and determine these interactions from the outcome of tests. Although DAs can be used to locate and detect interaction faults between neighboring factors, they are not well adapted for this kind of software testing. For example, some optimum DAs do not exist for fixed $t, k, v[21,22]$, but the optimum arrays for locating and detecting interaction faults between neighboring factors may exist. Moreover, combinatorial testing of neighboring factors only considers consecutive interactions, rather than arbitrary interactions. In an attempt to solve this problem, we propose a similar family of DAs, which we call consecutive detecting arrays (CDAs), in which the interactions between factors are considered to be ordered.

The remainder of this paper is organized as follows. The concept of CDAs is described in Section 2. The necessity for the existence of CDAs is also discussed in this section. In Section 3, we establish a general criterion for measuring the optimality of a CDA in terms of its size, and explore the equivalence between optimum CDAs and COAs with prescribed properties. Based on the equivalence outlined in Section 3, some constructions and existence results are presented in Section 4. Finally, Section 5 summarizes our concluding remarks.

\section{Consecutive Detecting Arrays}

This section explains the notion of CDAs. The necessity for the existence of CDAs is also discussed. To aid this discussion, a consecutive $t$-way interaction is defined below.

A consecutive $t$-way interaction is denoted as $T=\left\{\left(\left(i, x_{i}\right),\left(i+1, x_{i+1}\right), \cdots,\left(i+t-1, x_{i+t-1}\right)\right)\right\}$, where $1 \leq i \leq k-t+1, x_{r} \in V$ for $r=i, i+1, \cdots, i+t-1$. Obviously, there are a total of $(k-t+1) v^{t}$ consecutive $t$-way interactions for $k$ neighboring factors. To locate and detect interaction faults between neighboring factors, it is only necessary to identify the consecutive interaction faults from the outcomes of the tests. Thus, the notion of CDAs comes from modifying the notion of DAs.

Suppose that $A=\left(a_{i j}\right)\left(i \in I_{N}, j \in I_{k}\right)$ is a $\operatorname{CCA}(N ; t, k, v)$ over $V$. Write $C \mathcal{I}_{t}$ for the set of all consecutive $t$-way interactions of $A$. For any $\mathcal{T} \subseteq C \mathcal{I}_{t}$ with $|\mathcal{T}|=d$ and any $T \in C \mathcal{I}_{t}$, if we have

$$
\rho(A, T) \subseteq \rho(A, \mathcal{T}) \Leftrightarrow T \in \mathcal{T},
$$

then the array $A$ is called a $(d, t)$-consecutive detecting array, denoted by $(d, t)-\operatorname{CDA}(N ; k, v)$.

Clearly, a $(d, t)-\mathrm{DA}(N ; k, v)$ must be a $(d, t)-\operatorname{CDA}(N ; k, v)$, but the converse is not always true. It is straightforward that $T \in \mathcal{T}$ implies $\rho(A, T) \subseteq \rho(A, \mathcal{T})$. Hence, the condition $\rho(A, T) \subseteq \rho(A, \mathcal{T}) \Leftrightarrow$ $T \in \mathcal{T}$, is satisfied if $T \notin \mathcal{T} \Rightarrow \rho(A, T) \nsubseteq \rho(A, \mathcal{T})$. We will make extensive use of this simple fact in the following. As well as DAs, there are some admissible parameters for the existence of CDAs. We restrict our discussion to nontrivial parameters. As there are exactly $(k-t+1) v^{t}$ possible consecutive $t$-way interactions, we treat $(d, t)$-CDA only when $1 \leq d \leq(k-t+1) v^{t}$. When $k<t$ and $d>0$, no $(d, t)$-CDAs can exist. If $k=t$, we can form an array consisting of all $t$-tuples. Hence, we only treat cases with $k>t$. Finally, we require $v>1$ to avoid factors that take on unique levels. The following lemma states the necessary condition for the existence of CDAs.

Lemma 2.1 If $a(d, t)-C D A(N ; k, v)$ exists, then $d<v$. 
Proof. Suppose that $d \geq v$. We can form $v$ consecutive $t$-way interactions $\mathcal{T}=\left\{\left(\left(2, v_{2}\right),\left(3, v_{3}\right), \cdots\right.\right.$, $\left.\left.\left(t, v_{t}\right),(1, i)\right): i \in V\right\}$. Let $T$ be $\left(\left(2, v_{2}\right),\left(3, v_{3}\right), \cdots,\left(t, v_{t}\right),\left(t+1, v_{t+1}\right)\right)$. Clearly, $\rho(A, T) \subseteq \rho(A, \mathcal{T})$, but $T \notin \mathcal{T}$.

Lemma 2.2 Let $A$ be a $(d, t)$-CDA $(N ; k, v)$ that exists. Then, $A$ is also a $(s, t)$-CDA $(N ; k, v)$, where $1 \leq s \leq d-1$.

Proof. Let $\mathcal{T}$ be an arbitrary consecutive interactions set of cardinality $s$. Then, it holds that $\mathcal{T}_{1} \cap \mathcal{T}_{2}=$ $\mathcal{T}$, where $\left|\mathcal{T}_{1}\right|=\left|\mathcal{T}_{2}\right|=d$. Suppose that $\rho(A, T) \subseteq \rho(A, \mathcal{T})$ holds for any consecutive $t$-way interaction $T$. It can be easily shown that $\rho(A, T) \subseteq \rho\left(A, \mathcal{T}_{1}\right)$ and $\rho(A, T) \subseteq \rho\left(A, \mathcal{T}_{2}\right)$. As $A$ is a $(d, t)-\operatorname{CDA}(N ; k, v)$, we have $T \in \mathcal{T}_{1}$ and $T \in \mathcal{T}_{2}$ by the definition of CDAs. Thus, $T \in \mathcal{T}_{1} \cap \mathcal{T}_{2}=\mathcal{T}$. This completes the proof.

By definition, a $(d, t)$-CDA is actually a special CCA of strength $t$. The significance of using the CDA to generate test suites is that any set of $d$ consecutive $t$-way interaction faults can be determined from the outcomes. Further, if there are more than $d$ consecutive $t$-way interactions causing the faults, this can also be detected. For details, see the application of DAs in [7]. As the rows of a CDA stand for the number of tests, the CDA of minimum size when other parameters are fixed is of considerable interest. The minimum $N$ for which a $(d, t)-\operatorname{CDA}(N ; k, v)$ exists is referred to as the consecutive detecting arrays number $(\mathrm{CDAN})$, denoted by $(d, t)-\operatorname{CDAN}(k, v)$. A $(d, t)-\operatorname{CDA}(N ; k, v)$ with $N=(d, t)-\operatorname{CDAN}(k, v)$ is said to be optimum. In the next section, we derive a lower bound for the function $(d, t)$-CDAN $(k, v)$.

\section{Optimality Criterion and Combinatorial Description}

The objective of this section is to establish a lower bound on the size of $(d, t)-\operatorname{CDA}(N ; k, v)$ and explore the equivalence between optimum CDAs and a special class of COAs. We first establish a benchmark to measure the optimality of $(d, t)$-CDAs. The following result can be obtained by employing a similar proof as that of Lemma 2.1 in [24]. For completeness, we describe the proof in full.

Lemma 3.1 Suppose that $A$ is a $(1, t)-C D A(N ; k, v)$ with $t<k$. Then, $|\rho(A, T)| \geq 2$ for any consecutive $t$-way interaction $T$.

Proof. As a CDA is a special type of CCA, we have $|\rho(A, T)| \geq 1$. Thus, it suffices to show $|\rho(A, T)| \neq$ 1 for any consecutive $t$-way interaction $T$. If not, suppose that $\left(x_{1}, x_{2}, \ldots, x_{k}\right)$ is the unique row of $A$ that covers $T$. This row also covers $(k-t)$ consecutive $t$-way interactions other than $T$. It follows that there would be at least one $t$-way interaction $T^{\prime}(\neq T)$ such that $\rho(A, T) \subseteq \rho\left(A, T^{\prime}\right)$ under the assumption $t<k$. Thus, $A$ is not a $(1, t)-\operatorname{CDA}(N ; k, v)$.

The following lemma can be viewed as a generalization of Lemma 3.1. The proof is similar to that for Lemma 2.2 in [21]. We simply replace $t$-way interactions by consecutive $t$-way interactions and use the fact stated in Lemma 2.2. Thus, the proof is omitted here.

Lemma 3.2 Suppose that $A$ is a $(d, t)-C D A(N ; k, v)$ with $t<k$. Then, $|\rho(A, T)| \geq d+1$ for any consecutive $t$-way interaction $T$.

By applying Lemma 3.2, we have a lower bound on the function $(d, t)$-CDAN $(k, v)$. This serves as our benchmark for measuring the optimality of CDAs. 
Theorem 3.3 Let $t, k$, and $v$ be positive integers with $t<k$. Then,

$$
(d, t)-C D A N(k, v) \geq(d+1) v^{t} .
$$

Proof. Let $A$ be a $(d, t)-\operatorname{CDA}(N ; k, v)$ over $V$ with $t<k$ and $N=(d, t)-\operatorname{CDAN}(k, v)$. By definition, for the first $t$ columns $\{1,2, \cdots, t\}$, there exist exactly $v^{t} t$-way interactions of $A:\left\{\left(i, x_{i}\right): 1 \leq i \leq t, x_{i} \in V\right\}$. From Lemma 3.2, $|\rho(A, T)| \geq d+1$ for any consecutive $t$-way interaction $T$ of $A$. Therefore, $A$ must contain at least $(d+1) v^{t}$ rows. This means that $N=(d, t)-\operatorname{CDAN}(k, v) \geq(d+1) v^{t}$.

We call a $(d, t)-\operatorname{CDA}(N ; k, v)$ with $N=(d+1) v^{t}$ optimum. It is interesting that optimum CDAs have useful applications in software testing, because they contain minimum rows. In addition, optimum CDAs can be characterized in terms of a special class of COAs. To explore the combinatorial features of optimum CDAs, we need to introduce the notion of simple COAs. $\mathrm{A} \mathrm{COA}_{\lambda}(t, k, v)$ is simple if any $N \times(2 t-i)$ subarray consisting of two consecutive $t$ columns with $i$ columns in common contains each $(2 t-i)$-tuple at most once, where $0 \leq i \leq t-1$. From this definition, it is obvious that a simple $\operatorname{COA}_{\lambda}(t, k, v)$ can only exist if $\lambda \leq v$. In fact, a simple $\operatorname{COA}_{\lambda}(t, k, v)$ with $\lambda=1$ is a $\operatorname{COA}(t, k, v)$.

Example 3.1 The transpose of the following array is a simple $\mathrm{COA}_{3}(2,6,3)$ over $\mathbb{Z}_{3}$.

\begin{tabular}{lllllllllllllllllllllllllll|}
0 & 0 & 0 & 0 & 0 & 0 & 0 & 0 & 0 & 1 & 1 & 1 & 1 & 1 & 1 & 1 & 1 & 1 & 2 & 2 & 2 & 2 & 2 & 2 & 2 & 2 & 2 \\
0 & 0 & 0 & 1 & 1 & 1 & 2 & 2 & 2 & 0 & 0 & 0 & 1 & 1 & 1 & 2 & 2 & 2 & 0 & 0 & 0 & 1 & 1 & 1 & 2 & 2 & 2 \\
0 & 1 & 2 & 0 & 1 & 2 & 0 & 1 & 2 & 0 & 1 & 2 & 0 & 1 & 2 & 0 & 1 & 2 & 0 & 1 & 2 & 0 & 1 & 2 & 0 & 1 & 2 \\
0 & 2 & 1 & 2 & 1 & 0 & 1 & 0 & 2 & 2 & 1 & 0 & 1 & 0 & 2 & 0 & 2 & 1 & 1 & 0 & 2 & 0 & 2 & 1 & 2 & 1 & 0 \\
0 & 0 & 0 & 0 & 0 & 0 & 0 & 0 & 0 & 1 & 1 & 1 & 1 & 1 & 1 & 1 & 1 & 1 & 2 & 2 & 2 & 2 & 2 & 2 & 2 & 2 & 2 \\
0 & 1 & 2 & 0 & 1 & 2 & 0 & 1 & 2 & 0 & 1 & 2 & 0 & 1 & 2 & 0 & 1 & 2 & 0 & 1 & 2 & 0 & 1 & 2 & 0 & 1 & 2 \\
\hline
\end{tabular}

It is easy to check that any two consecutive columns contain each 2-tuple exactly twice, and each 4-tuple over $\mathbb{Z}_{3}$ from the two disjoint consecutive two-columns occurs at most once. For any two consecutive two-columns with one column in common, each 3-tuple occurs at most once.

The following theorem explores the equivalence between optimum CDAs and simple COAs.

Theorem 3.4 Suppose that $t$ and $k$ are two positive integers and $t<k$. Then, a simple $C O A_{d+1}(t, k, v)$ is equivalent to an optimum $(d, t)-C D A\left((d+1) v^{t} ; k, v\right)$.

Proof. $(\Rightarrow)$ Suppose that $A$ is a simple $\mathrm{COA}_{d+1}(t, k, v)$. Let $T$ be an arbitrary consecutive $t$-way interaction of $A$. Consider the set $\mathcal{T}=\left\{T_{1}, T_{2}, \ldots, T_{d}\right\}$ of arbitrary consecutive $t$-way interactions of cardinality $d$ such that $T \notin \mathcal{T}$. We only need to prove that $\rho(A, T) \nsubseteq \rho(A, \mathcal{T})$. As $A$ is a $\operatorname{COA}_{d+1}(t, k, v)$, $|\rho(A, T)|=d+1$ holds for any consecutive $t$-way interaction. If $\rho(A, T) \subseteq \rho(A, \mathcal{T})$, then there would be at least one $T_{j} \in \mathcal{T}$ such that $\left|\rho\left(A, T_{j}\right) \cap \rho(A, T)\right| \geq 2$. Suppose that the column indices of $T$ and $T_{j}$ have $i$ columns in common, where $0 \leq i \leq t-1$. Let $T^{\prime}$ be the interaction given by deleting the common elements of $T$ and $T_{j}$ from $T$. Because $T \neq T_{j}$, at least two rows in a certain $(2 t-i)$ columns would cover the interaction given by a concatenation of $T_{j}$ and $T^{\prime}$. This is inconsistent with the simple property of $A$.

$(\Leftarrow)$ Let $B$ be a $(d, t)-\operatorname{CDA}\left((d+1) v^{t} ; k, v\right)$ over the symbol set $V$. From Lemma 3.2, we know that $|\rho(A, T)|=d+1$ for any consecutive $t$-way interaction $T$, because $B$ contains precisely $(d+1) v^{t}$ rows. This indicates that each $t$-tuple occurs as a row exactly $(d+1)$ times in any consecutive $t$ columns of $B$. Thus, $B$ is a $\mathrm{COA}_{d+1}(t, k, v)$. The simple property of $B$ can easily be obtained from the definition of a CDA.

It is remarkable that a $(d, t)-\operatorname{CDA}(N ; k, v)$ cannot exist whenever $d<v$. This is consistent with the fact that a simple $\mathrm{COA}_{d+1}(t, k, v)$ can only exist if $d+1 \leq v$. 


\section{Construction and Existence of Optimum CDAs}

In this section, we use the equivalence characterization shown in Theorem 3.4 to construct a great number of optimum CDAs in terms of simple COAs. First, we describe the notion of super-simple orthogonal arrays (SSOA), which is analogous to the notion of simple COAs. We say that an $\mathrm{OA}_{\lambda}(t, k, v)$ is super-simple if any $(t+1)$ columns of the array contain every $(t+1)$-tuple of symbols as a row at most once. This is denoted by $\operatorname{SSOA}_{\lambda}(t, k, v)$. Clearly, an $\operatorname{SSOA}_{\lambda}(t, k, v)$ is a simple $\operatorname{COA}_{\lambda}(t, k, v)$, but the converse is not always true. It is remarkable that construction and existence of simple $\operatorname{COA}_{\lambda}(t, k, v)$ with $\lambda>1$ are considered unless otherwise specified, as we only treat a $(d, t)$-CDA with $d>0$ in this paper.

\subsection{Optimum CDAs from Orthogonal Arrays}

OAs are a highly structured family of arrays that were first introduced by Rao [20]. They are also important objects in combinatorics and experimental design. Over the past half a century, OAs have been the subject of considerable study. The following elegant result on the existence of $\mathrm{OA}(t, k, v)$ with $t \geq 3$ was derived by Bush [1].

Lemma 4.1 [1] If $q$ is a prime power and $t<q$, then an $O A(t, q+1, q)$ exists. Moreover, if $q \geq 4$ is a power of 2 , an $O A(3, q+2, q)$ exists.

Bush also established a powerful composite construction that serves to obtain new OAs from old ones. The derived array is formed by juxtaposing these known arrays.

Lemma 4.2 [2] If $O A\left(t, k, v_{i}\right)^{\prime}$ exists for all $1 \leq i \leq m$, then an $O A\left(t, k, \prod_{i=1}^{m} v_{i}\right)$ exists.

Lemmas 4.1 and 4.2 immediately give the following result.

Lemma 4.3 Suppose that $v=q_{1} q_{2} \cdots q_{s}$ is a standard factorization of $v$ into distinct prime powers. If $q_{i}>t$, then an $O A(t, k+1, v)$ exists, where $k=\min \left\{q_{i}: 1 \leq i \leq s\right\}$.

The following well-known result (see, for example, [5, 15]) can be obtained by zero-sum construction, i.e., for each of the $v^{t} t$-tuples over $\mathbb{Z}_{v}$, form a row vector of length $t+1$ by adjoining the negative of the sum of the elements in the first $t$ columns to the last column.

Lemma 4.4 An $O A(t, t+1, v)$ exists for any integer $v \geq 2$ and $t \geq 2$.

The existence of OAs with $t=3$ and $k=5,6$ was recently proved by Ji and Yin [17].

Lemma 4.5 [17] Let $v \geq 4$ be an integer. If $v \not \equiv 2(\bmod 4)$, then an $O A(3,5, v)$ exists.

Lemma 4.6 [17] Let $v$ be a positive integer that satisfies $\operatorname{gcd}(v, 4) \neq 2$ and $\operatorname{gcd}(v, 18) \neq 3$. Then, there is an $O A(3,6, v)$, and $O A(3,6,3 u)$ with $u \in\{5,7\}$ exists.

Our approach of constructing optimum CDAs from SSOAs is as follows. The process can be thought of as a modification of Construction 3.7 in [21].

Construction 4.7 If an $\operatorname{SSOA}_{\lambda}(t, k, v)$ exists, then a simple $\mathrm{COA}_{\lambda}(t, k+t-1, v)$ exists. 
Proof. Let $A$ be the given $\operatorname{SSOA}_{\lambda}(t, k, v)$ over the symbol set $V$ with column vectors $A_{1}, A_{2}, \cdots, A_{k}$. Write $A^{\prime}=\left(A_{1}, A_{2}, \cdots, A_{k}, A_{1}, A_{2}, \cdots, A_{t-1}\right)$. We claim that the array $A^{\prime}$ is a simple $\operatorname{COA}_{\lambda}(t, k+$ $t-1, v)$ over $V$, as desired. Clearly, it is a $\operatorname{COA}_{\lambda}(t, k, v)$ because any consecutive $t$ columns are the certain $t$ columns of $A$. It remains to prove that $A^{\prime}$ is simple. For any two consecutive $t$ columns $i, i+1, \cdots, i+t-1$ and $j, j+1, \cdots, j+t-1$, suppose that $|\{i, i+1, \cdots, i+t-1\} \cap\{j, j+1, \cdots, j+t-1\}|=l$ with $0 \leq l \leq t-1$, where $1 \leq i \leq k-1, i<j \leq k$. If $l=t-1$, then the consecutive $t+1$ columns are the certain $t+1$ columns of $A$. As $A$ is an $\operatorname{SSOA}_{\lambda}(t, k, v)$, each $(t+1)$-tuple occurs at most once. Hence, any $N \times(t+1)$ subarray consisting of the consecutive $t+1$ columns in $A^{\prime}$ contains each $(t+1)$-tuple at most once. This can be divided into two cases when $l=0$. If two disjoint consecutive $t$ columns are from the first $k$ columns of $A^{\prime}$, each $2 t$-tuple occurs at most once because each $(t+1)$-tuple occurs at most once in $A$. If two disjoint consecutive $t$ columns do not lie in the first $k$ columns of $A^{\prime}$ at the same time, then there are at least $(t+1)$ columns in the $2 t$ columns that lie in the columns of $A$. The super-simple property of $A$ guarantees that each $(t+1)$-tuple occurs at most once, and thus any $N \times 2 t$ subarray contains each $2 t$-tuple at most once. Similarly, if $1 \leq l \leq t-2$, we can prove that any $N \times(2 t-l)$ subarray is simple.

By Construction 3.7 in [21] and Construction [4.7, we have the following working method of construction.

Construction 4.8 If an $O A(t+1, k+1, v)$ exists, then a simple $\mathrm{COA}_{\lambda}(t, k+t-1, v)$ exists for any positive integer $\lambda \leq v$.

The following example illustrates the idea of Construction 4.8 .

Example 4.1 The transpose of the following array is an $\mathrm{OA}(3,4,2)$ over $\mathbb{Z}_{2}$.

\begin{tabular}{|llll|llll|}
\hline 0 & 0 & 0 & 0 & 1 & 1 & 1 & 1 \\
\hline 0 & 0 & 1 & 1 & 1 & 1 & 0 & 0 \\
0 & 1 & 0 & 1 & 1 & 0 & 1 & 0 \\
0 & 1 & 1 & 0 & 1 & 0 & 0 & 1 \\
\hline
\end{tabular}

We can derive two $\mathrm{OA}(2,3,2)$ over $\mathbb{Z}_{2}$ from this OA:

$$
A_{0}=\begin{array}{lll}
0 & 0 & 0 \\
0 & 1 & 1 \\
1 & 0 & 1 \\
1 & 1 & 0
\end{array} \quad A_{1}=\begin{array}{lll}
1 & 1 & 1 \\
1 & 0 & 0 \\
0 & 1 & 0 \\
0 & 0 & 1
\end{array}
$$

Write $M=\left(\begin{array}{c}A_{0} \\ A_{1}\end{array}\right)$. Using the above method of construction, we can obtain $M^{\prime}$ as follows. We write its transpose for simplicity.

$$
M^{\prime}=\left(\begin{array}{llllllll}
0 & 0 & 1 & 1 & 1 & 1 & 0 & 0 \\
0 & 1 & 0 & 1 & 1 & 0 & 1 & 0 \\
0 & 1 & 1 & 0 & 1 & 0 & 0 & 1 \\
0 & 0 & 1 & 1 & 1 & 1 & 0 & 0
\end{array}\right) .
$$

It can easily be checked that $M^{\prime}$ is a simple $\operatorname{COA}_{2}(2,4,2)$ over $\mathbb{Z}_{2}$.

If an $\mathrm{OA}(3, k+1, v)$ exists, then a simple $\mathrm{COA}_{\lambda}(2, k+1, v)$ exists by Construction 4.8 . In fact, we can improve the number of factors as follows. 
Construction 4.9 If an $O A(3, k+1, v)$ with $k>4$ exists, then a simple $C O A_{\lambda}(2,2 k+1, v)$ exists for any integer $\lambda \leq v$.

Proof. If an $\mathrm{OA}(3, k+1, v)$ exists, then an $\operatorname{SSOA}_{\lambda}(2, k, v)\left(A_{i}\right), x x x i \in I_{k}$ exists for any integer $\lambda \leq v$. Write

$$
A^{\prime}= \begin{cases}\left(A_{1}, A_{2}, \cdots, A_{k}, A_{1}, A_{3}, \cdots, A_{k-2}, A_{k}, A_{2}, A_{4}, \cdots, A_{k-3}, A_{k-1}, A_{1}\right) & \text { if } k \text { is odd } \\ \left(A_{1}, A_{2}, \cdots, A_{k}, A_{1}, A_{3}, \cdots, A_{k-3}, A_{k-1}, A_{2}, A_{4}, \cdots, A_{k-2}, A_{k}, A_{2}\right), & \text { if } k \text { is even. }\end{cases}
$$

It is easily checked that $A^{\prime}$ is the required simple COA.

Combining Theorem 3.4 and Constructions 4.8 and 4.9 with those known OAs given in the previous lemmas, it is possible to produce an infinite series of optimum CDAs.

Theorem 4.10 Let both $t \geq 2$ and $v \geq 2$ be integers. Then, an optimum $(d, t)-C D A\left((d+1) v^{t} ; 2 t, v\right)$ exists for any positive integer $d$ satisfying $d+1 \leq v$.

Proof. Under the given assumption, an $\mathrm{OA}(t+1, t+2, v)$ exists by Lemma4.4 Applying Construction 4.8 with this OA will produce a simple $\mathrm{COA}_{d+1}(t, 2 t, v)$, because $d+1 \leq v$. The conclusion follows from Theorem 3.4 with $k=2 t$.

Theorem 4.11 Let $q$ be a prime power. Then, an optimum $(d, t)-C D A\left((d+1) v^{t} ; t, q+t-1, q\right)$ exists for any positive integers $d$ and $t+1$ that are less than $q$. Moreover, if $q \geq 4$ is a power of 2 , then an optimum $(d, 2)-C D A\left((d+1) v^{2} ; 2 q+3, q\right)$ also exists.

Proof. Apply Theorem 3.4. The required simple COAs are obtained by Constructions 4.8 and 4.9 and Lemma 4.1 .

Theorem 4.12 Suppose that $v=q_{1} q_{2} \cdots q_{s}$ is a standard factorization of $v$ into distinct prime powers and $k=\min \left\{q_{i}: 1 \leq i \leq s\right\}$. If $q_{i}>t+1$ and $d+1 \leq v$, then there exists an optimum $(d, t)$ $C D A\left((d+1) v^{t} ; k+t-1, v\right)$.

Proof. By Lemma 4.3, we have an $\mathrm{OA}(t+1, k+1, v)$. Applying Construction 4.8 produces a simple $\mathrm{COA}_{d+1}(t, k+t-1, v)$ under the assumption $d+1 \leq v$. Hence, we can apply Theorem 3.4 to form the desired CDA.

Theorem 4.13 Let $v$ be a positive integer satisfying $\operatorname{gcd}(v, 4) \neq 2$ and $\operatorname{gcd}(v, 18) \neq 3$. Then, there exists an optimum $(d, 2)-C D A\left((d+1) v^{2} ; 11, v\right)$ for any positive integer $d$ with $d+1 \leq v$.

Proof. Apply Theorem 3.4. The required simple COAs are given by Construction 4.9 and Lemma 4.6 .

Theorem 4.14 Let $v \geq 4$ be an integer. If $v \neq \equiv 2(\bmod 4)$, then an optimum $(d, 2)-C D A\left((d+1) v^{2} ; 6, v\right)$ exists for any positive integer $d$ with $d+1 \leq v$.

Proof. Under the given assumption, an $\mathrm{OA}(3,5, v)$ exists by Lemma 4.5. An $\operatorname{SSOA}\left((d+1) v^{2} ; 4, v\right), A$ exists for any positive integer $d$ with $d+1 \leq v$. Let $A_{1}, A_{2}, A_{3}, A_{4}$ be the column vectors of $A$. The array $\left(A_{1}, A_{2}, A_{3}, A_{4}, A_{1}, A_{3}\right)$ is the desired optimum CDA. 


\subsection{Some more approaches for constructing simple COAs}

In this subsection, we present some more constructions of optimum CDAs in design theory. Let $A$ be a $\operatorname{COA}_{\lambda}(t, k, v)$ over the symbol set $V$. If the rows of $A$ can be partitioned into $\mu$ subarrays such that each has the simple property described above, then $A$ is termed a $\mu$-row-divisible $\operatorname{COA}_{\lambda}(t, k, v)$. Two simple COAs over the same symbol set are compatible if their superimposition constitutes a simple COA. A set of $w$ simple COAs over the same symbol set is termed compatible if all elements of the set are pairwise compatible. The notion of $\mu$-row-divisible and compatible OAs was first introduced in [21], where they were used to construct SSOAs. Here, we modify them to construct simple COAs.

Theorem 4.15 Let $v_{1}$ and $v_{2}$ be two positive integers such that $\mu$ compatible simple $C O A_{\eta}\left(t, k, v_{2}\right)^{\prime} s$ exist. Suppose that there are $r$ non-negative integers $m_{1}, m_{2}, \cdots, m_{r}$ and $2 r$ positive integers $\mu_{1}, \mu_{2}, \cdots, \mu_{r}$, $\lambda_{1}, \lambda_{2}, \cdots, \lambda_{r}$ such that the following two conditions are both satisfied:

1. $m_{1} \mu_{1}+m_{2} \mu_{2}+\cdots+m_{r} \mu_{r} \leq \mu$;

2. a $\mu_{i}$-row-divisible $\operatorname{COA}_{\lambda_{i}}\left(t, k, v_{1}\right)$ exists for $1 \leq i \leq r$.

Then, there exists a simple $C O A_{\eta\left(m_{1} \lambda_{1}+m_{2} \lambda_{2}+\cdots+m_{r} \lambda_{r}\right)}\left(t, k, v_{1} v_{2}\right)$.

Proof. Without loss of generality, we can assume that all the given row-divisible COAs are defined on the same symbol set $V$ (otherwise, we may take an appropriate permutation of the symbols). For each $i$ with $1 \leq i \leq r$, we form an $m_{i} \mu_{i}$-row-divisible $\operatorname{COA}_{m_{i} \lambda_{i}}\left(t, k, v_{1}\right)$ by taking $m_{i}$ copies of a $\mu_{i^{-}}$ row-divisible $\mathrm{COA}_{\lambda_{i}}\left(t, k, v_{1}\right)$. The superimposition of the resultant $r$ row-divisible OAs is then the $\left(m_{1} \mu_{1}+m_{2} \mu_{2}+\cdots+m_{r} \mu_{r}\right)$-row-divisible $\mathrm{OA}_{m_{1} \lambda_{1}+m_{2} \lambda_{2}+\cdots+m_{r} \lambda_{r}}\left(t, k, v_{1}\right)$, as desired.

By assumption, there exist $\mu$ compatible simple $\mathrm{COA}_{\eta}\left(t, k, v_{2}\right)^{\prime}$ s. Thus, the desired simple COAs can be obtained by a modification of the usual weighting method in design theory, as used in the proof of [14, Lemma 13] and [21, Construction 4.2].

By taking $r=1, m_{1}=1, \lambda_{1}=\lambda, v_{1}=v, \mu_{1}=\mu$, and $v_{2}=m$ in Theorem 4.15, we obtain the following corollary.

Corollary 4.16 Let $v, k$, and $t$ be integers satisfying $k \geq t \geq 2$. If a $\mu$-row-divisible $\operatorname{COA}_{\lambda}(t, k, v)$ and $\mu$ compatible simple $\mathrm{COA}_{\eta}(t, k, m)^{\prime}$ s all exist, then so does a simple $C O A_{\lambda \eta}(t, k, m v)$. In particular, if a simple $\mathrm{COA}_{\lambda}(t, k, v)$ and a simple $\mathrm{COA}_{\eta}(t, k, m)$ both exist, then so does a simple $\mathrm{COA}_{\lambda \eta}(t, k, m v)$.

\subsection{Existence spectrum of optimum CDAs with few factors for $t \in\{2,3\}$}

In this subsection, the existence of optimum CDAs with few factors is determined almost completely when $t \in\{2,3\}$. It is known that the derived array of an $\mathrm{OA}(t, k, v)$ is an $\mathrm{OA}(t-1, k-1, v)$. This simple fact is not always true for consecutive orthogonal arrays. The following example indicates this fact.

Example 4.2 The transpose of the following array is a COA $(4,6,2)$ over $\mathbb{Z}_{2}$.

\begin{tabular}{|llllllll|llllllll|}
\hline 0 & 0 & 0 & 0 & 0 & 0 & 0 & 0 & 1 & 1 & 1 & 1 & 1 & 1 & 1 & 1 \\
\hline 0 & 0 & 0 & 0 & 1 & 1 & 1 & 1 & 0 & 0 & 0 & 0 & 1 & 1 & 1 & 1 \\
0 & 0 & 1 & 1 & 0 & 0 & 1 & 1 & 0 & 0 & 1 & 1 & 0 & 0 & 1 & 1 \\
0 & 1 & 0 & 1 & 0 & 1 & 0 & 1 & 0 & 1 & 0 & 1 & 0 & 1 & 0 & 1 \\
0 & 0 & 1 & 1 & 0 & 0 & 1 & 1 & 1 & 1 & 0 & 0 & 1 & 1 & 0 & 0 \\
0 & 1 & 0 & 1 & 1 & 0 & 1 & 0 & 0 & 1 & 0 & 1 & 1 & 0 & 1 & 0 \\
\hline
\end{tabular}


The arrays $A_{0}$ and $A_{1}$ derived by deleting the last column are as follows.

$$
A(0)=\left[\begin{array}{llllllll}
0 & 0 & 0 & 0 & 1 & 1 & 1 & 1 \\
0 & 0 & 1 & 1 & 0 & 0 & 1 & 1 \\
0 & 1 & 0 & 1 & 0 & 1 & 0 & 1 \\
0 & 0 & 1 & 1 & 0 & 0 & 1 & 1 \\
0 & 1 & 0 & 1 & 1 & 0 & 1 & 0
\end{array}\right] \quad A(1)=\left[\begin{array}{llllllll}
0 & 0 & 0 & 0 & 1 & 1 & 1 & 1 \\
0 & 0 & 1 & 1 & 0 & 0 & 1 & 1 \\
0 & 1 & 0 & 1 & 0 & 1 & 0 & 1 \\
1 & 1 & 0 & 0 & 1 & 1 & 0 & 0 \\
0 & 1 & 0 & 1 & 1 & 0 & 1 & 0
\end{array}\right]
$$

Clearly, neither $A_{0}$ nor $A_{1}$ is a $\operatorname{COA}(3,5,2)$.

Example 4.2 tells us that the existence of a $\operatorname{COA}(t, k, v)$ does not imply the existence of a $\mathrm{COA}(t-$ $1, k-1, v)$. Moreover, deleting a certain column from a $\operatorname{COA}(t, k, v)$ does not guarantee it is a $\operatorname{COA}(t, k-1, v)$. Thus, we only construct simple COAs for each set of values $t, k, v$.

Theorem 4.17 An optimum $(d, 2)-C D A(k, v)$ with $k=3,4$ exists for any integer $d$ with $(d+1) \leq v$.

Proof. The existence of $\operatorname{SSOA}_{d+1}(2,3, v)$ is proved in [21]. Clearly, an SSOA is also a simple COA. A simple $\mathrm{COA}_{d+1}(2,4, v)$ can be obtained by taking $t=2$ in Theorem 4.10 . Applying Theorem 3.4 produces optimum CDAs, as desired.

Similarly, we can obtain the following result.

Theorem 4.18 An optimum $(d, 3)$-CDA $(4, v)$ exists for any integer $d$ with $(d+1) \leq v$.

The following theorem treats the case with $t=3, k=5,6$.

Theorem 4.19 An optimum $(d, 3)-C D A(k, v)$ with $k=5,6$ exists for any integer $d$ with $(d+1) \leq v$.

Proof. From Theorem 3.4, we only need to construct a simple $\mathrm{COA}_{d+1}(3,5, v)$ and $\operatorname{COA}_{d+1}(3,6, v)$ for any integer $d$ with $(d+1) \leq v$. We take $t=3$ in Construction 4.8 with an $\mathrm{OA}(4,5, v)$ to form arrays $A^{\prime}=\left(A_{1}, A_{2}, A_{3}, A_{4}, A_{1}\right)$ and $A^{\prime \prime}=\left(A_{1}, A_{2}, A_{3}, A_{4}, A_{1}, A_{2}\right)$, where $A_{i}$ is the $i$ th column of $A$. It is routine to check that $A^{\prime}$ and $A^{\prime \prime}$ are the simple $\operatorname{COA}_{d+1}(3,5, v)$ and $\operatorname{COA}_{d+1}(3,6, v)$, respectively.

By taking the array $\left(A_{1}, A_{2}, A_{3}, A_{4}, A_{1}\right)$ in Theorem 4.14 , we obtain the following result.

Theorem 4.20 Let $v \geq 4$ be an integer. If $v \not \equiv 2(\bmod 4)$, then an optimum $(d, 2)-C D A(5, v)$ exists for any integer $d$ with $(d+1) \leq v$.

For the completeness of existence for a $(d, 2)-\operatorname{CDA}(5, v)$, we consider the case $v=2,3$ or $v \geq 6$ and $v \equiv 2(\bmod 4)$. For $v \in\{2,3,6\}$, we have the following results.

Lemma 4.21 A 2-row-divisible $\mathrm{COA}_{3}(2,5,2)$ over $\mathbb{Z}_{2}$ exists.

Proof. Simply take the transpose of the following array with two partitions:

\begin{tabular}{|llllll|llllll|}
\hline 0 & 0 & 1 & 1 & 0 & 1 & 0 & 1 & 0 & 1 & 1 & 0 \\
0 & 0 & 1 & 0 & 1 & 1 & 1 & 0 & 1 & 1 & 0 & 0 \\
0 & 1 & 0 & 1 & 1 & 1 & 0 & 1 & 1 & 0 & 0 & 0 \\
0 & 0 & 1 & 1 & 1 & 0 & 1 & 1 & 0 & 0 & 0 & 1 \\
0 & 1 & 1 & 1 & 0 & 0 & 1 & 0 & 1 & 0 & 1 & 0 \\
\hline
\end{tabular}


Lemma 4.22 A simple $C O A_{v}(2,5, v)$ over $\mathbb{Z}_{v}$ exists for $v \in\{2,3,6\}$.

Proof. By Lemma 4.4 an $\mathrm{OA}(3,4, v)$ with column vectors $A_{1}, A_{2}, A_{3}, A_{4}$ exists. Write $A^{\prime}=\left(A_{1}, A_{2}\right.$, $\left.A_{3}, A_{4}, A_{1}\right) . A$ is the required simple OA for $v=2,3,6$.

Lemma 4.23 A simple $\operatorname{COA}_{\lambda}(2,5, v)$ over $\mathbb{Z}_{v}$ exists for $(\lambda, v) \in\{(2,3),(2,6),(4,6),(1,6)\}$.

Proof. The existence of an $\operatorname{SSOA}_{2}(2,5, v)$ for $v=3,6$ is proved in [4]. This implies the existence of simple $\mathrm{COA}_{2}(2,5,3)$ and $\mathrm{COA}_{2}(2,5,6)$. A simple $\mathrm{COA}_{4}(2,5,6)$ can be obtained by Corollary 4.16. The ingredient $\mathrm{COA}_{2}(2,5,2)$ is given by Lemma 4.22, $\mathrm{A} \operatorname{COA}(2,5,6)$ is constructed using the array $\left(A_{1}, A_{2}, A_{3}, A_{1}, A_{3}\right)$, where $A_{i}$ is the $i$ th column of an $\mathrm{OA}(2,3,6)$.

Lemma 4.24 A 2-row-divisible $C O A_{\lambda}(2,5,6)$ over $\mathbb{Z}_{6}$ with $\lambda \in\{3,5,9\}$ exists.

Proof. Let $A$ and $B$ be a 2-row-divisible $\mathrm{COA}_{3}(2,5,2)$ with the partition $A_{1}, A_{2}$ and a simple $\mathrm{COA}_{3}(2$, 5,3 ), respectively. Take $V=\mathbb{Z}_{2} \times Z_{3}$. Over $V$, we form a $9 \cdot 6^{2} \times 5$ array $\bar{A}$ as follows. For each row $\left(a_{i 1}, a_{i 2}, \cdots, a_{i 5}\right)$ of $A_{i}$ and each row $\left(b_{h 1}, b_{h 2}, \cdots, b_{h k}\right)$ of $B$ for $i=1,2$, include the row $\left\{\left(a_{i 1}, b_{h 1}\right),\left(a_{i 2}, b_{h 2}\right), \cdots,\left(a_{i k}, b_{h k}\right)\right\}$ in $\bar{A}$ as a row. It is easy to check that the resultant array $\bar{A}$ is a 2 row-divisible $\mathrm{COA}_{9}(2,5,6)$. A 2-row-divisible $\mathrm{COA}_{\lambda}(2,5,6)$ over $\mathbb{Z}_{6}$ with $\lambda=3,5$ can be obtained by the juxtaposition of an $\mathrm{OA}(2,5,6)$ and a simple $\mathrm{COA}_{2}(2,5,6)$ or a simple $\mathrm{COA}_{4}(2,5,6)$, respectively.

We now determine the existence for a simple $\operatorname{COA}_{\lambda}(2,5, v)$ with $v \equiv 2(\bmod 4)$. Write $v=4 t+2=$ $2(2 t+1)$, where $t \geq 2$. To apply the corollary in Subsection 4.2, we need compatible COAs, which can be obtained using the simple argument in [21].

Lemma 4.25 Let $v=2 t+1$ be an integer with $t \geq 2$. If $2 t+1 \neq 3 u$ with $\operatorname{gcd}(u, 6)=1$, then $v$ compatible $\operatorname{COA}(2,5, v)^{\prime}$ s exist. Moreover, if $v=2 t+1=3 u$ with $u \neq 1$ and $\operatorname{gcd}(u, 6)=1$, the $u$ compatible $\operatorname{COA}(2,5, u)^{\prime}$ s exist.

Proof. Under the given assumption and Lemma 4.6, an OA $(3,6, v)$ exists. By Construction 3.7 in [21], the $v$ derived arrays given by deleting the first column form $v$ compatible COAs. The second assertion can be proved in a similar way to the first statement.

Lemma 4.26 Let $v=4 t+2$ be an integer with $t \geq 2$. If $2 t+1 \neq 3 u$ with $\operatorname{gcd}(u, 6)=1$, then a simple $\mathrm{COA}_{\lambda}(2,5, v)$ exists for any integer $\lambda \leq v$.

Proof. From Lemmas 4.21 and 4.22, a $\mu_{i}$-row-divisible $\mathrm{OA}_{\lambda_{i}}(2,5,2)$ exists for $i=1,2$. Apply Theorem 4.15 with $v_{1}=2, v_{2}=2 t+1,\left(\mu_{1}, \lambda_{1}\right)=(1,2)$, and $\left(\mu_{2}, \lambda_{2}\right)=(2,3)$. It remains to show that the system of equations

$$
\left\{\begin{array}{l}
2 m_{1}+3 m_{2}=\lambda \\
m_{1}+2 m_{2} \leq 2 t+1
\end{array}\right.
$$

is solvable for non-negative integers $m_{1}$ and $m_{2}$ for any given $\lambda$ with $\lambda \leq 4 t+2$.

It now turns out that

$$
\left(m_{1}, m_{2}\right)=\left\{\begin{array}{l}
\left(\frac{\lambda}{2}, 0\right), \text { if } \lambda \text { is even }, \\
\left(\frac{\lambda-3}{2}, 1\right), \text { if } \lambda \text { is odd }
\end{array}\right.
$$

is one solution of the above system of equations. 
Lemma 4.27 Let $v=6 u$ be an integer with $u \neq 1$ and $\operatorname{gcd}(u, 6)=1$. Then, a simple $\operatorname{COA}_{\lambda}(2,5, v)$ exists for any integer $\lambda \leq v$ except for $\lambda=v-1$.

Proof. From Lemmas 4.22 4.24, we know that a simple $\operatorname{COA}_{\lambda}(2,5,6)$ with $\lambda \in\{1,2,4,6\}$ and a 2row-divisible $\operatorname{COA}_{\lambda}(2,5,6)$ with $\lambda=3,5,9$ all exist. For any given $\lambda$ with $\lambda \leq 6 u$ and $\lambda \notin\{6 u-$ $1,6 u-3\}$, we write $h=\lfloor\lambda / 6\rfloor$ and $\varepsilon=\lambda-6 h \in\{0,1, \cdots, 5\}$. Then,

$$
\left\{\begin{array}{l}
h \leq u, \text { if } \varepsilon=0, \\
h \leq u-1, \text { if } \varepsilon=1,2,4, \\
h \leq u-2, \text { if } \varepsilon=3,5 .
\end{array}\right.
$$

Apply Theorem 4.15 with $v_{1}=6, v_{2}=u,\left(\mu_{1}, \lambda_{1}\right)=(1,6)$, and

$$
\left(\mu_{2}, \lambda_{2}\right)=\left\{\begin{array}{l}
(1,1), \text { if } \varepsilon=1 \\
(1,2), \text { if } \varepsilon=2 \\
(2,3), \text { if } \varepsilon=3 \\
(1,4), \text { if } \varepsilon=4 \\
(2,5), \text { if } \varepsilon=5
\end{array}\right.
$$

It can easily be checked that the system of equations

$$
\left\{\begin{array}{l}
6 m_{1}+\lambda_{2} m_{2}=\lambda \\
m_{1}+\mu_{2} m_{2} \leq u
\end{array}\right.
$$

has one solution with non-negative integers:

$$
\left(m_{1}, m_{2}\right)=\left\{\begin{array}{l}
(h, 0), \text { if } \varepsilon=0 ; \\
(h, 1), \text { if } \varepsilon=1,2,3,4,5 .
\end{array}\right.
$$

For $\lambda=6 u-3$, we apply Theorem 4.15 with the ingredients $\operatorname{COA}_{6}(3,5,6)$ and 2-row-divisible $\mathrm{OA}_{9}(3,5,6)$.

Combining Theorem 3.4 with the results in Lemmas 4.22 4.27, we have the following results.

Theorem 4.28 An optimum $(d, 2)-C D A(5, v)$ exists for any positive integer $d+1 \leq v$, except possibly where

$$
\begin{aligned}
& \text { 1. }(d, v) \in\{(2,6),(4,6)\} ; \\
& \text { 2. } v=6 u \text { and } d=v-2 \text {, where } u \neq 1 \text { and } \operatorname{gcd}(u, 6)=1 \text {. }
\end{aligned}
$$

\section{Concluding Remarks}

Detecting arrays are used to generate test suites for locating and detecting interaction faults between factors. For practical software testing, there may only be interactions between neighboring factors. Although DAs can be used to locate and detect interaction faults between neighboring factors, they are not well adapted for this kind of software testing. This paper has introduced the notion of consecutive detecting arrays to solve this problem. Consecutive detecting arrays are of interest in generating software test suites to cover any consecutive $t$-way component interactions and locate interaction faults between neighboring factors. In this paper, a general lower bound on the size of $(d, t)-\operatorname{CDA}(N ; k, v)$ has been established. The equivalence between the optimum $(d, t)-\operatorname{CDA}\left((d+1) v^{t} ; k, v\right)$ and a simple 
$\mathrm{COA}_{d+1}(t, k, v)$ was explored in Theorem 3.4. Based on this equivalence, a great number of optimum CDAs that satisfy the lower bound were obtained by constructing simple COAs. The existence spectrum of $(d, t)-\operatorname{CDA}\left((d+1) v^{t} ; k, v\right)$ with few factors for $t=2,3$ was almost completely determined. Future studies will focus on finding new techniques for constructing simple COAs and deriving more results with large numbers of factors.

\section{References}

[1] K. A. Bush, Orthogonal arrays of index unity, Ann. Math. Stat., 23: 426-434, 1952.

[2] K. A. Bush, A generalization of the theorem due to MacNeish, Ann. Math. Stat., 23: 293-295, 1952.

[3] M. Chateauneuf, C. J. Colbourn and D. L. Kreher, Covering arrays of strength three, Des. Codes Cryptogr., 16: 235-242, 1999.

[4] Y. H. Chen, Constructions of Optimal Detecting Arrays of Degree 5 and Strength 2, Master Thesis, Soochow University, 2011.

[5] C. J. Colbourn and J. H. Dinitz, The CRC Handbook of Combinatorial Designs. CRC Press, Boca Raton, FL, 2007.

[6] M. Chateauneuf and D. L. Kreher, On the state of strength-three covering arrays, J. Combin. Des., 10: 217-238, 2002.

[7] Colbourn C. J., McClary D. W.: Locating and detecting arrays for interaction faults. J. Comb. Optim. 15: 17-48, 2008.

[8] C. J. Colbourn, S. S. Martirosyan, T. V. Trung and R. A. Walker II, Roux-type constructions for covering arrays of strengths three and four, Des. Codes Cryptogr., 41: 33-57, 2006.

[9] C. J. Colbourn, Combinatorial aspects of covering arrays, LeMatematiche (Catania) 58: 121167, 2004.

[10] C. J. Colbourn, Strength two covering arrays: Existence tables and projection, Discrete Math. 308: 772-786, 2008.

[11] A. P. Godbole, M. V. Koutras, and F. S. Milienos, Binary consecutive covering arrays, Annals of the Institute of Statistical Mathematics , 63(3): 559?584, 2011.

[12] A. P. Godbole, M. V. Koutras and F. S. Milienos, Consecutive covering arrays and a new randomness test, Journal of Statistical Planning and Inference , 140(5): 1292-1305, 2010.

[13] A. Hartman and L. Raskin, Problems and algorithms for covering arrays, Discrete Math., 284: 149-156, 2004.

[14] S. Hartman, On simple and supersimple transversal designs, J. Comb. Des., 8: 311-322, 2000.

[15] A. S. Hedayat, N. J. A. Slone and J. Stufken, Orthogonal Arrays, Springer, New York, 1999.

[16] J. T. Jimenez and I. I. Marquez, Covering arrays of strength three from extended permutation vectors, Des. Codes Cryptogr., 86(11): 2629-2643, 2018. 
[17] L. Ji and J. Yin, Constructions of new orthogonal arrays and covering arrays of strength three, $J$. Combin. Theory (A), 117: 236-247, 2010.

[18] D. R. Kuhn and M. J. Reilly, An investigation of the applicability of design of experiments to software testing, Proceedings of the 27th NASA/ IEEE Software Engineering Workshop, NASA Goddard Space Flight Center, 91-95,2002.

[19] D. R. Kuhn and D. R. Wallance, Software fault interaction and implication for software testing, IEEE Transactions on Software Engineering , 30(6): 1-4, 2004.

[20] C. R. Rao, Factorial experiments derivable from combinatorial arrangements of arrays, Supplement to the Journal of the Royal Statistical Society, 9: 128-139,1947.

[21] C. Shi, Y. Tang and J. X. Yin, The equivalence between optimal detecting arrays and supersimple OAs, Des. Codes Cryptogr., 62: 131-142,2012.

Des. Codes Cryptogr.,

[22] C. Shi and J. X. Yin, Existence of super-simple $\left.\mathrm{OA}_{\lambda}(3,5, v)\right]^{\prime}$ s, Des. Codes Cryptogr., 72: 369380, 2014.

[23] G. Tzanakis, L. Moura, D. Panario and B. Stevens, Covering arrays from m-sequences and character sums, Des. Codes Cryptogr., 85(3): 437456, 2017.

[24] Y. Tang and J. X. Yin, Detecting arrays and their optimality. Acta Math. Sin., Engl. Ser., 27: 2309-2318, 2011.

[25] Z. Y. Wang, C. H. Nie, B. W. Xu and L. Shi, Optimal Test Suite Generation Methods for Neighbor Factors Combinatorial Testing (in chinese), Chinese Journal of Computers, 30(2): 200-211, 2007. 